BMJ Open Diabetes

Research \& Care

\title{
Association between non-alcoholic fatty liver disease-associated hepatic fibrosis and bone mineral density in postmenopausal women with type 2 diabetes or impaired glucose regulation
}

\author{
Xiaopeng Zhu, ${ }^{1,2}$ Hongmei Yan, ${ }^{1,2}$ Xinxia Chang, ${ }^{1,2}$ Mingfeng Xia, ${ }^{1,2}$ \\ Linshan Zhang, ${ }^{1,2}$ Liu Wang, ${ }^{1,2}$ Xiaoyang Sun, ${ }^{1,2}$ Xinyu Yang, ${ }^{1,2}$ Xin Gao, ${ }^{1,2}$ \\ Hua Bian (1) ${ }^{1,2}$
}

To cite: Zhu X, Yan H, Chang X, et al. Association between non-alcoholic fatty liver disease-associated hepatic fibrosis and bone mineral density in postmenopausal women with type 2 diabetes or impaired glucose regulation. BMJ Open Diab Res Care 2020;8:e000999. doi:10.1136/ bmjdrc-2019-000999

Additional material is published online only. To view, please visit the journal online (http://dx.doi.org/10.1136/ bmjdrc-2019-000999).

Received 22 0ctober 2019 Revised 2 April 2020 Accepted 22 June 2020

Check for updates

(C) Author(s) (or their employer(s)) 2020. Re-use permitted under CC BY-NC. No commercial re-use. See rights and permissions. Published by BMJ.

${ }^{1}$ Department of Endocrinology and Metabolism, Zhongshan Hospital Fudan University, Shanghai, China

${ }^{2}$ Fudan Institute for Metabolic Disease, Fudan University, Shanghai, China

Correspondence to Dr Hua Bian; zhongshan_bh@126.com and Dr Xin Gao;

zhongshan_endo@126.com

\section{ABSTRACT}

Introduction To evaluate the association of non-alcoholic fatty liver disease (NAFLD)-associated hepatic fibrosis with bone mineral density (BMD) in postmenopausal women with type 2 diabetes mellitus (T2DM) or impaired glucose regulation (IGR).

Research design and methods Two cohorts including 46 subjects with biopsy-proven NAFLD and 445 subjects with proton magnetic resonance spectrum-proven NAFLD were enrolled in this study. All subjects were postmenopausal women with T2DM or IGR. BMD at the lumbar spine L1-L4 and hip was measured using dual-energy X-ray absorptiometry. NAFLD fibrosis stage and NAFLD fibrosis score were used to evaluate the severity of liver fibrosis. Results In subjects with liver biopsy-proven NAFLD, BMD (T-score, Z-score and BMD value) in the advanced fibrosis group were significantly lower than that in the non-advanced fibrosis group $(p<0.05)$. Fibrosis stage was negatively associated with T-score, Z-score and BMD value after adjusting for age, body mass index (BMI) and fasting plasma glucose (FPG). Additionally, fibrosis stage was independently associated with T-score, Z-score and $B M D$ value after adjusting for age, BMl and FPG. These results were validated in a large cohort of 445 subjects. Additionally, bone metabolism-associated factors, including calcium and phosphate, were associated with liver fibrosis, indicating that bone metabolism may play a critical role in the association between liver fibrosis and BMD. Mechanically, parathyroid hormone and biomarkers of bone formation (osteocalcin and procollagen type 1 $\mathrm{N}$-terminal propeptide) and bone resorption (procollagen type I carboxy terminal peptide $\beta$ special sequence) were increased in subjects with advanced liver fibrosis than in subjects without advanced liver fibrosis, indicating that liver fibrosis decreased BMD probably via increasing bone turnover.

Conclusions NAFLD-associated hepatic fibrosis was negatively associated with decreased BMD in postmenopausal women with T2DM or IGR. Liver fibrosis decreased BMD probably via increasing bone turnover. Severe liver fibrosis may represent high risk for osteoporosis in postmenopausal women with T2DM or IGR.

\section{Significance of this study}

What is already known about this subject?

- There is an association of non-alcoholic fatty liver disease (NAFLD) with bone mineral density (BMD) in children, adult males and females; however, the relation between NAFLD-associated hepatic fibrosis and BMD remains unknown.

What are the new findings?

- NAFLD-associated hepatic fibrosis was negatively associated with decreased BMD in postmenopausal women with type 2 diabetes mellitus (T2DM) or impaired glucose regulation (IGR).

- Bone metabolism-associated factors, including calcium and phosphate, were associated with liver fibrosis.

- Biomarkers of bone formation and resorption were increased in subjects with advanced liver fibrosis than in subjects without advanced liver fibrosis.

- Liver fibrosis decreased BMD probably via increasing bone turnover.

How might these results change the focus of research or clinical practice?

- Severe NAFLD-associated liver fibrosis may represent high risk for osteoporosis in postmenopausal women with T2DM or IGR; screening of reduced BMD in this population may be needed.

\section{INTRODUCTION}

Non-alcoholic fatty liver disease (NAFLD), characterized by excessive fat deposition in hepatocytes without overt alcohol consumption or other liver injury, is becoming a major public health issue worldwide. ${ }^{1} \mathrm{~A}$ recent study shows that the global prevalence of NAFLD is up to $25.2 \%$ and the proportion of fibrosis progression in non-alcoholic steatohepatitis (NASH) is $40.8 \% .^{2}$ NAFLD, the pathology 
spectrum of which ranges from simple steatosis to NASH and cirrhosis, is considered as a hepatic manifestation of metabolic syndrome. ${ }^{3}$ In addition, NAFLD is a multiple system disease, which is usually accompanied by extrahepatic complications, such as type 2 diabetes mellitus (T2DM), cardiovascular disease, chronic kidney disease, osteoporosis and neoplasm. ${ }^{4-7}$ It seems that NAFLD probably plays a significant role in the pathogenesis of other organs or systems.

Osteoporosis is defined as a bone mineral density (BMD) $>2.5$ standard deviations (SDs) below the young normal mean at the spine, hip or mid-radius by World Health Organization (WHO). Osteoporosis is also a significant public health issue worldwide like NAFLD, especially in elderly population. ${ }^{8}$ Previous studies illustrated that there was a complex network between adipose tissue, liver and bone. ${ }^{9} 10$ Consistent with these findings, several studies demonstrated there was an association of NAFLD with BMD in children, adult males and females. ${ }^{11-20}$ However, the relation between NAFLD and BMD remains controversial. Our previous study including 1659 subjects found that there was a negative correlation between liver fat content (LFC) and BMD in middle-aged and elderly Chinese men and postmenopausal women. ${ }^{21}$ However, the method of NAFLD diagnosis in these studies is ultrasonography, ${ }^{12}{ }^{14-16}{ }^{18-20}$ which is subjective and is greatly influenced by operator. Liver biopsy is still the gold standard to diagnose liver inflammation and fibrosis at present. Furthermore, whether NAFLDassociated hepatic fibrosis is correlated with BMD remains unknown. Previous study showed chronic inflammation and cirrhosis were risk factors for reduced BMD. ${ }^{22}$ Therefore, we hypothesized that NAFLD-associated hepatic fibrosis was correlated with low BMD. Previous studies showed that postmenopausal women had a high risk of reduced BMD, and patients with T2DM had a high risk of NAFLD and fracture. ${ }^{23-25}$ Therefore, we chose postmenopausal women with T2DM or impaired glucose regulation (IGR) as our target population.

In this study, we evaluated the association between NAFLD-associated liver fibrosis and BMD (T-score, Z-score and BMD value) in 46 postmenopausal women with biopsy-proven NAFLD and validated this correlation in a large cohort of 445 postmenopausal women with proton magnetic resonance spectrum $\left({ }^{1} \mathrm{H}\right.$-MRS)-proven NAFLD. We also explored the role of bone metabolismassociated factors in NAFLD-associated liver fibrosis, in order to uncover the potential mechanism of low BMD in patients with NAFLD-associated liver fibrosis.

\section{METHODS}

Study subjects

Two cohorts including 46 subjects with biopsy-proven NAFLD (mean age $58 \pm 5$ years) and 445 subjects with ${ }^{1}$ H-MRS-proven NAFLD (mean age $64 \pm 9$ years) from Zhongshan Hospital, Fudan University, were included in this study. All subjects had IGR (impaired fasting glucose or impaired glucose tolerance) or T2DM. The inclusion criteria for participants were as follows: i) postmenopausal women; ii) fatty liver diagnosed by biopsy or ${ }^{1} \mathrm{H}-$ MRS; iii) no known acute or chronic disease other than obesity, hypertension, diabetes, osteoporosis or NAFLD, based on history and physical examination. Exclusion criteria included i) autoimmune, viral or drug-induced liver disease; ii) excessive use of alcohol ( $>20 \mathrm{~g} /$ day for men, $>10 \mathrm{~g} /$ day in women ${ }^{26}$ ); iii) celiac disease.

\section{Dual-energy X-ray absorptiometry measurements of BMD}

T-score, Z-score and BMD value at the lumbar spine L1L4 (lumbar total) and hip (femoral neck, inter, troch and total) were measured using dual-energy X-ray absorptiometry (DXA) (Hologic Discovery DXA system).

\section{Evaluation of liver steatosis, inflammation and fibrosis in subjects with biopsy}

Liver biopsy was performed to determine the severity of liver steatosis, inflammation and fibrosis. NASH was diagnosed by the joint presence of steatosis, ballooning and lobular inflammation. ${ }^{27}$ NAFLD activity score (NAS) and NAFLD fibrosis stage (S) were applied to evaluate the severity of NAFLD. ${ }^{28-30}$ NAS, ranging from 0 to 8 , consists of steatosis (0-3), lobular inflammation (0-3) and ballooning (0-2). Briefly, steatosis was scored as $0(<5 \%), 1(5 \%-33 \%), 2(34 \%-66 \%)$ and $3(>66 \%)$; lobular inflammation (200× field) was scored as 0 (no foci), 1 (<2 foci), 2 (2-4 foci) and 3 ( $>4$ foci); ballooning was scored as 0 (none), 1 (few balloon cells) and 2 (many cells/prominent ballooning) ${ }^{28} \mathrm{~S}$ comprises four stages, including S0 (none), S1 (perisinusoidal or periportal), S2 (perisinusoidal and portal/periportal), S3 (bridging fibrosis) and S4 (cirrhosis). ${ }^{28}$

\section{Evaluation of liver steatosis, inflammation and fibrosis in subjects without biopsy}

${ }^{1}$ H-MRS was used to quantify LFC. Serum alanine aminotransferase (ALT) level was applied as a simple marker of liver inflammation. NAFLD fibrosis score (NFS) was calculated to evaluate the severity of hepatic fibrosis. ${ }^{31}$ $\mathrm{NFS}=-1.675+0.037 \times$ age (years $)+0.094 \times$ body mass index (BMI, $\left.\mathrm{kg} / \mathrm{m}^{2}\right)+1.13 \times$ impaired fasting glucose/diabetes (yes $=1$, no $=0)+0.99 \times$ aspartate aminotransferase (AST) / ALT ratio-0.013×platelet $\left(10^{9} / \mathrm{L}\right)-0.66 \times$ albumin $(\mathrm{g} /$ dL). ${ }^{31}$ NFS $\geq 0.676$ was considered as advanced fibrosis. ${ }^{31}$

\section{Anthropometric and laboratory measurements}

All subjects performed routine anthropometric measurements, serum biochemical examinations and history collections. A standard meal glucose tolerance test was performed in patients with a history of T2DM; an oral glucose tolerance test (OGTT, $75 \mathrm{~g}$ glucose) was performed in patients without a history of T2DM. Standing height, waist circumference and bodyweight were measured without shoes and outer clothing, and the BMI was calculated as weight $(\mathrm{kg})$ divided by standing height squared $\left(\mathrm{m}^{2}\right)$. Blood samples were collected after a fasting period of at least 12 hours. Total serum 
cholesterol (TC), triglycerides (TG), high-density lipoprotein cholesterol (HDL-ch), low-density lipoprotein cholesterol (LDL-ch), calcium, phosphate, uric acid, parathyroid hormone (PTH) and liver enzyme (ALT, AST, gamma-glutamyl transferase $(\gamma-G T))$ levels were measured using a model 7600 automated bio-analyzer (Hitachi, Tokyo, Japan). The fasting plasma glucose (FPG) and the 2-hour OGTT glucose following an OGTT or a standard meal were measured through glucose oxidase method. The fasting C-peptide and 2-hour OGTT C-peptide were measured via electrochemiluminescence. Hemoglobin A1c (HbA1c) was measured via high performance liquid chromatography. Plasma osteocalcin and 25-hydroxyvitamin D $(25(\mathrm{OH}) \mathrm{D})$ were measured using electrochemiluminescence immunoassay.

\section{Statistical analysis}

All statistical analyses were carried out using SPSS software V.20.0 (SPSS, Chicago, Illinois, USA). All data were texted for normality and homogeneity of variance. Normally distributed data are shown as the mean \pm SD, whereas non-normally distributed data are shown as median followed by the 25th and 75th percentiles. For normal and non-normal distribution of data, the unpaired Student's t-test and the Mann-Whitney U test were used to compare mean values between two groups, respectively. Pearson's correlation coefficient was calculated for normal distribution of data and Spearman's rank correlation coefficient for non-normal distribution of data. Multiple linear regression analysis was performed to investigate the independent associated factors with BMD. All tests were two-sided, and $\mathrm{p}<0.05$ was considered as statistical significance.

\section{RESULTS}

Clinical characteristics of subjects with biopsy-proven NAFLD A total of 46 subjects (mean age $58 \pm 5$ years) were divided into non-advanced fibrosis and advanced fibrosis groups based on the fibrosis stage ( $\mathrm{S} \geq 2$ is considered as advanced fibrosis). ${ }^{28}$ The proportion of T2DM in the advanced fibrosis group was higher than that in the non-advanced fibrosis group $(100.0 \%$ vs $78.9 \%, \mathrm{p}<0.05$, table 1). No significant difference was found in serum

Table 1 Clinical characteristics of the subjects

\begin{tabular}{|c|c|c|c|c|c|c|}
\hline & Subjects with biop & proven NAFLD & & Subjects with ${ }^{1} \mathrm{r}$ & RS-proven NAFLD & \\
\hline & $\begin{array}{l}\text { Total } \\
(n=46)\end{array}$ & $\begin{array}{l}S<2 \\
(n=19)\end{array}$ & $\begin{array}{l}S \geq 2 \\
(n=27)\end{array}$ & $\begin{array}{l}\text { Total } \\
(n=445)\end{array}$ & $\begin{array}{l}\text { Fibrosis score } \\
<0.676(n=305)\end{array}$ & $\begin{array}{l}\text { Fibrosis score } \\
\geq 0.676(n=140)\end{array}$ \\
\hline Age (years) & $58 \pm 5$ & $57 \pm 5$ & $59 \pm 6$ & $64 \pm 9$ & $62 \pm 8$ & $70 \pm 9^{\# \#}$ \\
\hline $\mathrm{BMI}\left(\mathrm{kg} / \mathrm{m}^{2}\right)$ & $26.3 \pm 4.0$ & $25.8 \pm 3.8$ & $26.7 \pm 4.2$ & $24.6 \pm 3.9$ & $23.8 \pm 3.4$ & $26.1 \pm 4.3^{\# \#}$ \\
\hline Waist circumference $(\mathrm{cm})$ & $91.3 \pm 8.5$ & $92.8 \pm 9.5$ & $90.2 \pm 7.8$ & $89.0 \pm 11.1$ & $86.4 \pm 9.8$ & $94.4 \pm 11.6^{\# \#}$ \\
\hline $\mathrm{FPG}(\mathrm{mmol} / \mathrm{L})$ & $6.5 \pm 1.7$ & $6.4 \pm 1.4$ & $6.6 \pm 1.9$ & $8.2 \pm 3.1$ & $8.3 \pm 3.1$ & $8.0 \pm 3.0$ \\
\hline 2-hour PG (mmol/L) & $13.9 \pm 3.9$ & $12.9 \pm 4.2$ & $14.8 \pm 3.6$ & $16.8 \pm 5.3$ & $17.2 \pm 5.3$ & $15.7 \pm 5.2$ \\
\hline $\mathrm{HbA1c}(\%)$ & $7.3 \pm 1.5$ & $7.0 \pm 1.2$ & $7.4 \pm 1.7$ & $9.3 \pm 2.1$ & $9.3 \pm 2.0$ & $9.3 \pm 2.3$ \\
\hline $\begin{array}{l}\text { Omin OGTT C-peptide } \\
(\mathrm{ng} / \mathrm{mL})\end{array}$ & $2.83(2.29-3.60)$ & $2.73(2.26-3.29)$ & $2.96(2.41-3.80)$ & $1.82(1.08-2.38)$ & $1.78(0.95-2.38)$ & $1.84(1.48-2.65)$ \\
\hline $\begin{array}{l}\text { 2-hour OGTT C-peptide } \\
\text { (ng/mL) }\end{array}$ & $10.45(7.50-13.55)$ & $9.11(6.76-11.3)$ & $12.0(8.0-15.3)$ & 4.65 (3.14-4.69) & 4.65 (3.14-6.39) & $4.54(3.28-7.94)$ \\
\hline $\mathrm{TC}(\mathrm{mmol} / \mathrm{L})$ & $4.79 \pm 1.10$ & $4.56 \pm 1.25$ & $4.95 \pm 0.98$ & $4.79 \pm 1.25$ & $4.91 \pm 1.33$ & $4.53 \pm 1.02^{\# \#}$ \\
\hline Triglyceride (mmol/L) & $1.93(1.43-2.31)$ & $1.96(1.56-3.06)$ & $1.90(1.40-2.27)$ & $1.47(1.08-2.13)$ & 1.46 (1.09-2.18) & $1.50(1.14-2.11)$ \\
\hline $\mathrm{HDL}-\mathrm{ch}(\mathrm{mmol} / \mathrm{L})$ & $1.13 \pm 0.25$ & $1.11 \pm 0.23$ & $1.14 \pm 0.27$ & $1.22 \pm 0.37$ & $1.24 \pm 0.38$ & $1.18 \pm 0.35$ \\
\hline LDL-ch (mmol/L) & $2.86 \pm 0.90$ & $2.72 \pm 0.91$ & $2.97 \pm 0.90$ & $2.71 \pm 0.96$ & $2.73 \pm 0.97$ & $2.66 \pm 0.95$ \\
\hline Calcium (mmol/L) & $2.30 \pm 0.13$ & $2.26 \pm 0.10$ & $2.33 \pm 0.14$ & $2.22 \pm 0.11$ & $2.23 \pm 0.10$ & $2.20 \pm 0.11^{\# \#}$ \\
\hline Phosphate (mmol/L) & $1.31 \pm 0.16$ & $1.30 \pm 0.16$ & $1.32 \pm 0.16$ & $1.29 \pm 0.19$ & $1.32 \pm 0.17$ & $1.21 \pm 0.20^{\# \#}$ \\
\hline Magnesium (mmol/L) & $0.87 \pm 0.10$ & $0.87 \pm 0.07$ & $0.86 \pm 0.11$ & $0.85 \pm 0.09$ & $0.85 \pm 0.08$ & $0.83 \pm 0.10$ \\
\hline Osteocalcin (ng/mL) & $17.8 \pm 9.4$ & $17.4 \pm 6.2$ & $18.0 \pm 11.2$ & $16.0 \pm 8.2$ & $15.7 \pm 7.0$ & $16.5 \pm 10.3$ \\
\hline 25(OH)D (nmol/L) & $41.0 \pm 19.1$ & $36.4 \pm 19.2$ & $43.7 \pm 18.9$ & $36.0 \pm 15.7$ & $36.9 \pm 15.5$ & $34.2 \pm 16.0$ \\
\hline PTH (pg/mL) & $42.3 \pm 14.4$ & $42.8 \pm 14.7$ & $42.0 \pm 14.5$ & $37.2 \pm 16.1$ & $35.6 \pm 14.6$ & $40.9 \pm 18.6^{\# \#}$ \\
\hline ALT (U/L) & $72(46-96)$ & $52(27-95)$ & $78(60-98)^{\star}$ & $16(11-26)$ & $17(12-27)$ & $13(10-22)$ \#\# \\
\hline AST (U/L) & $45(30-60)$ & $39(25-58)$ & $53(35-68)$ & $18(15-25)$ & $18(15-24)$ & $18(15-26)$ \\
\hline$\gamma-\mathrm{GT}(\mathrm{U} / \mathrm{L})$ & $52(31-75)$ & $39(29-89)$ & $57(41-75)$ & $24(17-40)$ & $24(16-39)$ & $25(17-47)$ \\
\hline LFC $\left(\%,{ }^{1} \mathrm{H}-\mathrm{MRS}\right)$ & $41.6(20.9-55.5)$ & $27.6(19.3-55.9)$ & $43.0(30.7-55.1)$ & $14.9(8.4-25.7)$ & $14.8(8.7-26.6)$ & $15.4(6.7-23.7)$ \\
\hline Diabetes (\%) & $91.3 \%$ & $78.9 \%$ & $100.0 \%$ * & $92.6 \%$ & $92.1 \%$ & $95.7 \%$ \\
\hline
\end{tabular}

${ }^{*} \mathrm{P}<0.05$ vs $\mathrm{S}<2 ;{ }^{\# \#} \mathrm{p}<0.01$ vs fibrosis score $<0.676$

ALT, alanine aminotransferase; AST, aspartate aminotransferase; BMI, body mass index; FPG, fasting plasma glucose; $\gamma$-GT, gamma-glutamyl transferase; HbA1c, hemoglobin A1c; HDL-ch, high-density lipoprotein-cholesterol; ${ }^{1} \mathrm{H}-\mathrm{MRS}$, proton magnetic resonance spectrum; LDL-ch, low-density lipoprotein-cholesterol; LFC, liver fat content; NAFLD, nonalcoholic fatty liver disease; OGTT, oral glucose tolerance test; $25(\mathrm{OH}) \mathrm{D}, 25$-hydroxyvitamin D; PG, postprandial plasma glucose; PTH, parathyroid hormone; S, fibrosis stage in pathology; TC, total cholesterol. 
Table 2 The difference of BMD in the different fibrosis groups in postmenopausal women

\begin{tabular}{|c|c|c|c|c|c|c|}
\hline & \multicolumn{3}{|c|}{ Subjects with biopsy-proven NAFLD ( $n=46)$} & \multicolumn{3}{|c|}{ Subjects with ${ }^{1} H$-MRS-proven NAFLD $(n=445)$} \\
\hline & $S<2(n=19)$ & $S \geq 2(n=27)$ & $P$ value & $\begin{array}{l}\text { NFS }<0.676 \\
(n=305)\end{array}$ & $\begin{array}{l}\text { NFS } \geq 0.676 \\
(n=140)\end{array}$ & $P$ value \\
\hline \multicolumn{7}{|l|}{ Femoral neck } \\
\hline T-score & $-1.2(-1.6--0.3)$ & $-1.4(-1.9--0.7)$ & 0.237 & $-1.5(-2.2--0.7)$ & $-1.9(-2.6--1.2)$ & $<0.001$ \\
\hline Z-score & $0.1(-0.5-1.0)$ & $-0.1(-0.7-0.9)$ & 0.384 & $-0.1(-0.7-0.6)$ & $-0.1(-0.8-0.5)$ & 0.991 \\
\hline BMD value & $0.719(0.667-0.806)$ & $0.689(0.643-0.771)$ & 0.372 & $0.690(0.609-0.786)$ & $0.634(0.580-0.717)$ & $<0.001$ \\
\hline \multicolumn{7}{|l|}{ Femoral troch } \\
\hline T-score & $-0.4(-0.8-0.0)$ & $-0.5(-1.0-0.0)$ & 0.468 & $-0.9(-1.6--0.2)$ & $-1.3(-1.9--0.7)^{\#}$ & $<0.001$ \\
\hline Z-score & $0.6(0.1-0.7)$ & $0.3(-0.2-1)$ & 0.541 & $-0.0(-0.5-0.8)$ & $-0.1(-0.6-0.5)^{\# \#}$ & 0.376 \\
\hline BMD value & $0.668(0.619-0.697)$ & $0.652(0.610-0.705)$ & 0.639 & $0.613(0.549-0.682)$ & $0.570(0.512-0.631)^{\# \#}$ & $<0.001$ \\
\hline \multicolumn{7}{|l|}{ Femoral inter } \\
\hline T-score & $0.3(-0.4-0.5)$ & $-0.6(-1.2-0.0)^{\star}$ & 0.018 & $-0.7(-1.4--0.1)$ & $-1.2(-1.9--0.3)^{\#}$ & $<0.001$ \\
\hline Z-score & $0.6(-0.4-0.8)$ & $0.1(-0.3-0.9)$ & 0.375 & $0.2(-0.5-0.7)$ & $0.1(-0.6-0.9)$ & 0.985 \\
\hline BMD value & $1.118(1.007-1.152)$ & $1.029(0.913-1.095)$ & 0.059 & $0.993(0.892-1.103)$ & $0.921(0.830-1.040)^{\#}$ & $<0.001$ \\
\hline \multicolumn{7}{|l|}{ Femoral total } \\
\hline T-score & $-0.2(-0.9-0.5)$ & $-0.7(-1.4--0.1)^{*}$ & 0.078 & $-0.8(-1.6--0.2)$ & $-1.4(-2.1--0.6)$ & $<0.001$ \\
\hline Z-score & $0.6(-0.5-1.2)$ & $0.1(-0.5-1.1)^{*}$ & 0.093 & $0.2(-0.5-0.9)$ & $0.2(-0.5-0.8)^{\#}$ & 0.582 \\
\hline BMD value & $0.921(0.834-1.012)$ & $0.865(0.769-0.932)^{*}$ & 0.090 & $0.846(0.761-0.935)$ & $0.779(0.705-0.869)^{\#}$ & $<0.001$ \\
\hline \multicolumn{7}{|l|}{ Lumbar total } \\
\hline T-score & $-1.3(-1.7--0.2)$ & $-0.9(-1.9--0.3)$ & 0.747 & $-1.5(-2.3--0.5)$ & $-1.6(-2.6--0.4)$ & 0.380 \\
\hline Z-score & $-0.2(-1.7--0.2)$ & $0.4(-0.5-0.9)$ & 0.845 & $0.1(-0.7-1)$ & $0.5(-0.5-1.5)$ & 0.010 \\
\hline BMD value & $0.947(0.864-1.031)$ & $0.947(0.841-1.010)$ & 0.616 & $0.922(0.824-1.032)$ & $0.896(0.800-1.038)$ & 0.267 \\
\hline
\end{tabular}

${ }^{*} \mathrm{P}<0.05$ vs $\mathrm{S}<2$, after adjusting for age and $\mathrm{BMI} ;{ }^{\#} \mathrm{p}<0.05$ and ${ }^{\# \#} \mathrm{p}<0.01$ vs NFS $<0.676$, after adjusting for age and BMI.

BMD, bone mineral density; BMI, body mass index; ${ }^{1} \mathrm{H}-\mathrm{MRS}$, proton magnetic resonance spectrum; NAFLD, non-alcoholic fatty liver disease; NFS, NAFLD fibrosis score; $\mathrm{S}$, fibrosis stage in pathology.

liver enzymes (AST and $\gamma$-GT), FPG, 2-hour postprandial plasma glucose (PG), HbA1c, TG, HDL-ch, LDL-ch, TC, osteocalcin, 25( $\mathrm{OH}) \mathrm{D}$ and LFC between the two groups.

\section{BMD in different stage of liver fibrosis in subjects with biopsy-proven NAFLD}

We next compared the BMD (T-score, Z-score and BMD value) in groups divided by fibrosis stage based on liver biopsy. In subjects with liver biopsy, only T-score (femoral inter) in the advanced fibrosis group was significantly lower than that in the non-advanced fibrosis group $(\mathrm{p}<0.05$, table 2$)$. In addition, subjects in the advanced fibrosis group had a trend toward lower T-score on hip at femoral neck, troch and total (table 2). After adjusting for age and BMI, T-score (femoral inter) and T-score (femoral total) in the advanced fibrosis group were significantly lower than those in the non-advanced fibrosis group (all $\mathrm{p}<0.05$, table 2 ). The Z-score is a value that compares with the age-matched normal. Z-score (femoral total) in the advanced fibrosis group was significantly lower than that in the non-advanced fibrosis group after adjusting for age and BMI ( $<<0.05$, table 2$)$. We also analyzed the value of BMD in non-advanced fibrosis and advanced fibrosis groups. BMD value (femoral total) in the advanced fibrosis group was significantly lower than that in the non-advanced fibrosis group after adjusting for age and BMI $(\mathrm{p}<0.05$, table 2$)$.
We further explored BMD in the non-NASH and NASH groups. No significant difference was found in T-score, Z-score and BMD value between the two groups (online supplementary table 1 ).

\section{Association of multiple metabolic factors with BMD or liver fibrosis in subjects with biopsy-proven NAFLD}

The association of multiple metabolic factors with BMD (T-score, Z-score and BMD value) or liver fibrosis in postmenopausal women with biopsy-proven NAFLD was determined. Serum HbAlc, phosphate, osteocalcin, PTH, $\beta$-CTX and PINP levels were negatively correlated with T-score, Z-score and BMD value after adjusting for BMI, age and FPG (all $\mathrm{p}<0.05$, online supplementary table 2 , online supplementary table 3 and online supplementary table 4). In addition, fibrosis stage was negatively associated with T-score, Z-score and BMD value on hip at femoral inter and total after adjusting for BMI, age and FPG (figure $1 \mathrm{~A}$ and B, online supplementary table 2, online supplementary table 3 and online supplementary table 4).

The association between multiple metabolic factors relative to bone metabolism and liver fibrosis in postmenopausal women with liver biopsy was explored. However, no association between metabolic factors (except PTH) relative to bone metabolism and liver fibrosis was found in these subjects (table 3 ). 
A

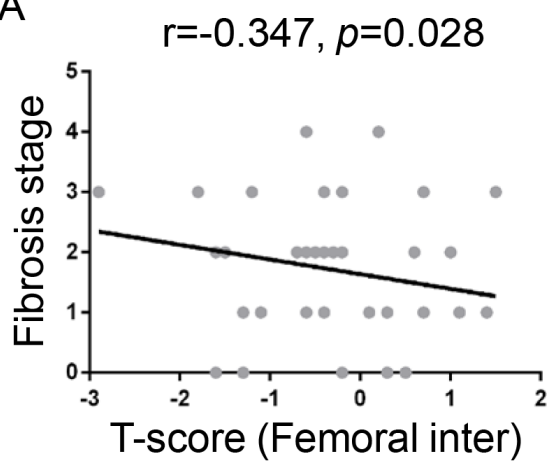

B

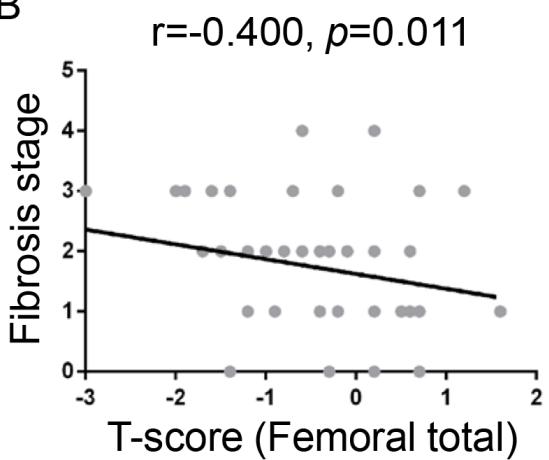

C

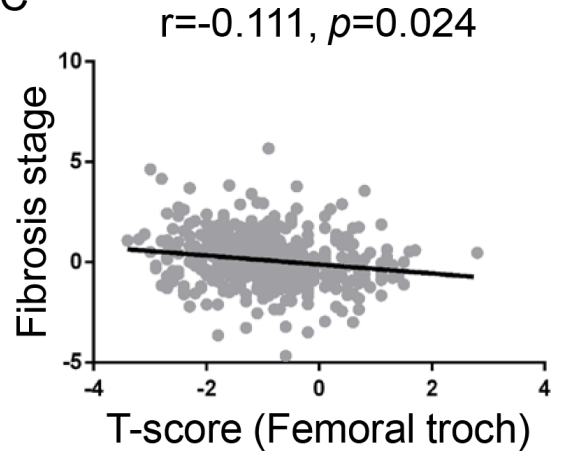

Figure 1 Association between liver fibrosis and bone mental density in postmenopausal women. (A) The association between fibrosis stage and T-score (femoral inter). (B) The association between fibrosis stage and T-score (femoral total). (C) The association between NFS and T-score (femoral troch). NAFLD, non-alcoholic fatty liver disease; NFS, NAFLD fibrosis score.

We further used multiple linear regression analysis to investigate the independent association between liver fibrosis and BMD (T-score, Z-score and BMD value) in postmenopausal women with liver biopsy. After adjusting for age, BMI and FPG, age and fibrosis stage were independently associated with T-score (femoral inter) (table 4); age, BMI and fibrosis stage were independently associated with T-score (femoral total) (table 4). Additionally, BMI, FPG and fibrosis stage were independently associated with Z-score (femoral inter) (online supplementary table 5); BMI and fibrosis stage were independently associated with Z-score (femoral total) after adjusting for age, BMI and FPG (online supplementary table 5). The linear regression was also performed for BMD value. Age, BMI and fibrosis stage were independently associated with BMD value on hip at femoral inter and total after adjusting for age, BMI and FPG (online supplementary table 6).

Table 3 The association between multiple metabolic parameters and liver fibrosis in postmenopausal women after adjusting for BMI, age and FPG

\begin{tabular}{|c|c|c|c|c|}
\hline & \multicolumn{2}{|c|}{$\begin{array}{l}\text { Subjects with biopsy- } \\
\text { proven NAFLD }(n=46)\end{array}$} & \multicolumn{2}{|c|}{$\begin{array}{l}\text { Subjects with }{ }^{1} \mathrm{H}-\mathrm{MRS}- \\
\text { proven NAFLD }(\mathrm{n}=445)\end{array}$} \\
\hline & $\begin{array}{l}\text { Fibrosis } \\
\text { stage }\end{array}$ & $P$ valve & NFS & $P$ valve \\
\hline Calcium & 0.111 & 0.507 & -0.193 & $<0.001$ \\
\hline Phosphate & 0.317 & 0.052 & -0.190 & $<0.001$ \\
\hline Magnesium & -0.083 & 0.619 & -0.125 & 0.016 \\
\hline PTH & -0.138 & 0.037 & 0.189 & $<0.001$ \\
\hline Osteocalcin & 0.155 & 0.353 & 0.117 & 0.024 \\
\hline $25(\mathrm{OH}) \mathrm{D}$ & -0.065 & 0.697 & -0.138 & 0.008 \\
\hline$\beta-$ CTX & 0.257 & 0.119 & 0.077 & 0.138 \\
\hline PINP & 0.305 & 0.062 & 0.114 & 0.028 \\
\hline
\end{tabular}

BMI, body mass index; $\beta$-CTX, procollagen type I carboxy terminal peptide $\beta$ special sequence; FPG, fasting plasma glucose; ${ }^{1} \mathrm{H}-\mathrm{MRS}$, proton magnetic resonance spectrum; NAFLD, non-alcoholic fatty liver disease; NFS, NAFLD fibrosis score; 25(OH)D, 25-hydroxyvitamin D; PINP, procollagen type $1 \mathrm{~N}$-terminal propeptide; PTH, parathyroid hormone.

\section{Clinical characteristics of subjects with ${ }^{1} \mathrm{H}$-MRS-proven NAFLD}

We validated the results above in a large cohort of 445 postmenopausal women (mean age $64 \pm 9$ years) with ${ }^{1}$ H-MRS-proven NAFLD. The subjects were divided into non-advanced fibrosis and advanced fibrosis groups based on the NFS (NFS $\geq 0.676$ was considered as advanced fibrosis). Age, BMI, waist circumference, and serum PTH level in the advanced fibrosis group were higher than those in the non-advanced fibrosis group (all $\mathrm{p}<0.01$, table 1 ), whereas serum total cholesterol, calcium, and phosphate in the advanced fibrosis group were lower than those in the non-advanced fibrosis group (all $\mathrm{p}<0.01$, table 1 ). No significant difference was found in serum FPG, 2-hour PG, HbAlc, TG, HDL-ch, LDL-ch, osteocalcin, 25(OH)D, LFC and the proportion of diabetes between the two groups.

\section{BMD in the different stage of liver fibrosis in subjects with ${ }^{1} \mathrm{H}$-MRS-proven NAFLD}

In agreement with the result from subjects with liver biopsy, T-score on hip at femoral neck, troch, inter and total in the advanced fibrosis group were significantly lower than those in the non-advanced fibrosis group (all $\mathrm{p}<0.001$, table 2). No significant difference was found in T-score (lumbar total) between the two groups. After adjusting for age and BMI, T-score on hip at femoral troch and inter in the advanced fibrosis group were still significantly lower than those in the non-advanced fibrosis group (all $\mathrm{p}<0.05$, table 2 ). Additionally, Z-score on hip at femoral troch and total in the advanced fibrosis group were significantly lower than those in the non-advanced fibrosis group after adjusting for age and BMI (all $\mathrm{p}<0.05$, table 2). BMD value on hip at femoral troch, inter and total in the advanced fibrosis group were significantly lower than those in the non-advanced fibrosis group after adjusting for age and BMI (all p<0.05, table 2).

\section{Association of multiple metabolic factors with BMD or liver fibrosis in subjects with ${ }^{1} \mathrm{H}-\mathrm{MRS}$-proven NAFLD}

The association of multiple metabolic factors with BMD (T-score, Z-score and BMD value) or liver fibrosis in 
Table 4 Multiple linear regression analysis for the association between multiple metabolic parameters and BMD in postmenopausal women (T-score)

\begin{tabular}{|c|c|c|c|c|c|c|c|c|c|}
\hline & \multicolumn{4}{|c|}{$\begin{array}{l}\text { Subjects with biopsy-proven NAFLD } \\
(n=46)\end{array}$} & & \multicolumn{4}{|c|}{$\begin{array}{l}\text { Subjects with }{ }^{1} \mathrm{H} \text {-MRS-proven NAFLD } \\
(\mathrm{n}=445)\end{array}$} \\
\hline & $\beta$ value & Std $\beta$ value & T value & $P$ value & & $\beta$ value & $\begin{array}{l}\text { Std } \beta \\
\text { value }\end{array}$ & $\mathrm{T}$ value & $P$ value \\
\hline \multicolumn{5}{|c|}{ Femoral inter: T-score } & \multicolumn{5}{|c|}{ Femoral troch: T-score } \\
\hline Age & -0.094 & -0.517 & -3.896 & $<0.001$ & Age & -0.036 & -0.326 & -5.435 & $<0.001$ \\
\hline Fibrosis stage & -0.263 & -0.298 & -2.284 & 0.028 & BMI & 0.095 & 0.367 & 6.525 & $<0.001$ \\
\hline \multicolumn{5}{|c|}{ Femoral total: T-score } & \multicolumn{5}{|c|}{ Femoral total: T-score } \\
\hline Age & -0.088 & -0.478 & -3.719 & 0.001 & Age & -0.047 & -0.383 & -6.503 & $<0.001$ \\
\hline BMI & 0.077 & 0.328 & 2.554 & 0.015 & BMl & 0.096 & 0.335 & 6.061 & $<0.001$ \\
\hline Fibrosis stage & -0.303 & -0.340 & -2.691 & 0.011 & NFS & -0.164 & -0.176 & -2.822 & 0.005 \\
\hline
\end{tabular}

For subjects with biopsy-proven NAFLD, age, BMI and FPG were adjusted.

For subjects with ${ }^{1} \mathrm{H}-\mathrm{MRS}$-proven NAFLD, age, BMI, FPG, TC, HDL-ch and LDL-ch were adjusted.

$\mathrm{BMD}$, bone mineral density; BMI, body mass index; FPG, fasting plasma glucose; $\mathrm{HDL}-\mathrm{ch}$, high-density lipoprotein-cholesterol; ${ }^{1} \mathrm{H}-\mathrm{MRS}$, proton magnetic resonance spectrum; LDL-ch, low-density lipoprotein-cholesterol; NAFLD, non-alcoholic fatty liver disease; NFS, NAFLD fibrosis score; TC, total cholesterol.

postmenopausal women with ${ }^{1} \mathrm{H}$-MRS-proven NAFLD was determined. NFS was negatively associated with T-score (femoral troch), Z-score (femoral troch), BMD value (femoral troch) and BMD value (femoral total) after adjusting for BMI, age and FPG (figure 1C, online supplementary table 2 , online supplementary table 3 and online supplementary table 4 ). Additionally, serum osteocalcin, PTH, $\beta$-CTX, PINP and HDL-ch were also negatively associated with T-score, Z-score and BMD value, whereas serum ALT was positively associated with T-score and Z-score (all $\mathrm{p}<0.05$, online supplementary table 2, online supplementary table 3 and online supplementary table 4) after adjusting for BMI, age and FPG.

We also explored the association between multiple metabolic factors relative to bone metabolism and liver fibrosis in this cohort. Serum calcium, phosphate, magnesium and 25(OH)D levels were negatively associated with NFS after adjusting for BMI, age and FPG (table 3).

We further performed multiple linear regression analysis to investigate the independent association between liver fibrosis and BMD in subjects with ${ }^{1} \mathrm{H}$-MRS-proven NAFLD. In line with previous results, age, BMI and NFS were independently associated with T-score on hip at femoral troch and total (table 4) after adjusting for age, BMI, FPG, TC, HDL-ch and LDL-ch. Additionally, BMI and NFS were independently associated with Z-score on hip at femoral troch and total; BMI, FPG and NFS were independently associated with Z-score (femoral inter) after adjusting for age, BMI, FPG, TC, HDL-ch and LDL-ch (online supplementary table 5). Age, BMI and NFS were independently associated with BMD value on hip at femoral inter, troch and total after adjusting for age, BMI, FPG, TC, HDL-ch and LDL-ch (online supplementary table 6 ).

\section{DISCUSSION}

In this study, based on liver biopsy, we found that NAFLD-associated hepatic fibrosis had an independent association with low BMD (T-score, Z-score and BMD value) in postmenopausal women with T2DM or IGR. The results were verified in a large cohort of postmenopausal women with T2DM or IGR, showing that NAFLD-associated liver fibrosis evaluated by NFS had an independent association with low BMD (T-score, Z-score and BMD value) in postmenopausal women with T2DM or IGR. Additionally, bone metabolismassociated factors, including calcium and phosphate, were associated with liver fibrosis, indicating that bone metabolism probably plays a critical role in the association between liver fibrosis and BMD. Based on these results, we tried to investigate the potential mechanism underlying the relation between BMD and liver fibrosis. We found that PTH and biomarkers of bone formation (osteocalcin and PINP) and bone resorption ( $\beta$-CTX) were increased in subjects with advanced liver fibrosis compared with those in subjects without advanced liver fibrosis, indicating that liver fibrosis decreased BMD probably via increasing bone turnover. These data suggest that severe liver fibrosis may represent high risk for osteoporosis in postmenopausal women with T2DM or IGR.

Few studies had focused on the relation of liver inflammation and fibrosis with BMD based on liver histology. Recent study including 35 children with histologically proven NAFLD has showed that children with NASH had a lower lumbar Z-score as well as a lower whole body Z-score, compared with those with simple steatosis. ${ }^{17}$ Another study enrolling 38 children with obesity with biopsy-proven NAFLD and 38 children with obesity without NAFLD demonstrated that children with obesity with NAFLD had lower Z-score than 
subjects without NAFLD, and children with NASH had a lower Z-score than subjects with NAFLD but without NASH. ${ }^{13}$ These two studies were conducted in children and had not reported the association between liver fibrosis and BMD. Recent study has revealed significant liver fibrosis diagnosed via liver transient elastography was independently associated with decreased BMD in patients with NAFLD. ${ }^{32}$ However, liver transient elastography is not the gold standard for evaluation of liver fibrosis. In current study, we found that advanced liver fibrosis diagnosed by the gold standard, liver biopsy, was independently associated with decreased BMD in postmenopausal women with NAFLD with T2DM or IGR.

Due to the small size (46 subjects) of postmenopausal women with liver biopsy, we validated the results in another large cohort of 445 postmenopausal women with ${ }^{1}$ H-MRS-proven NAFLD. As expected, advanced liver fibrosis was also independently associated with decreased BMD in this large cohort.

Although there is an association between NAFLD fibrosis and decreased BMD, the potential molecular mechanisms underlying this relation are still unknown. Study showed that NAFLD, especially NASH, may participate in the pathophysiology of decreased BMD via the systemic release of multiple pro-inflammatory, procoagulant, pro-oxidant and profibrogenic mediators and/ or via the direct effect on hepatic and systemic insulin resistance. ${ }^{33}$ In present study, $25(\mathrm{OH}) \mathrm{D}$, calcium, phosphate and magnesium were negatively associated with NFS. This might be because the synthesis of $25(\mathrm{OH}) \mathrm{D}$ was reduced in liver fibrosis condition. Reduced $25(\mathrm{OH})$ D led to decreased calcium absorption, which resulted in decreased BMD. As to phosphate, the explanation was that LFC was decreased as liver fibrosis became more serious, and phosphate was positively associated with LFC. ${ }^{22}$ Therefore, phosphate was negatively associated with liver fibrosis.

Osteocalcin, a major non-collagenous hydroxyapatitebinding protein produced by osteoblasts, which is considered as a biomarker of bone formation, ${ }^{34}{ }^{35}$ was reported to be negative associated with BMD both in premenopausal women with obesity and in postmenopausal women. ${ }^{36}{ }^{37}$ PINP is also a biomarker of bone formation. In current study, we found that osteocalcin and PINP were positively associated with NFS. $\beta$-CTX, a biomarker of bone resorption, had a trend toward positively associated with fibrosis stage/NFS in this study. Therefore, we speculated that liver fibrosis increased bone turnover, thus leading to decreased BMD. Moreover, in this study, increased PTH could be explained by compensation for low calcium, and increased PTH indirectly supported the increased bone turnover.

Previous studies showed that age was negatively associated with BMD, and BMI was positively associated with BMD. ${ }^{38}{ }^{39}$ We also found the same results in the present study. Study showed that patients with diabetes were prone to develop osteoporosis. ${ }^{40}$ In current study, we adjusted for FPG and found that NAFLD fibrosis was still an independent risk factor for decreased BMD.

Several limitations exist in this study. First, this is a cross-sectional study, and the association of NAFLD fibrosis with BMD should be further tested and verified in a prospective study. Second, the sample size of subjects with liver biopsy is small, the results of which are limited. Third, most subjects in this study have NAFLD accompanied by T2DM or IGR. Although we adjusted for FPG, the conclusion should be further investigated in nondiabetes population.

In conclusion, NAFLD-associated hepatic fibrosis was independently associated with decreased BMD in postmenopausal women with T2DM or IGR. Severe liver fibrosis may represent high risk for osteoporosis in postmenopausal women with T2DM or IGR.

Acknowledgements The authors would like to thank Zhongshan Hospital for supporting this study.

Contributors HB and XG designed the study. XZ, LZ, LW, XS and XY collected the data. $\mathrm{HY}, \mathrm{XC}$ and $\mathrm{MX}$ assessed the data. $\mathrm{XZ}$ and $\mathrm{HB}$ performed statistical analyses, and wrote the manuscript. XG edited the manuscript. All authors have approved the final version of the article.

Funding This work was supported by the financial support of National Natural Science Foundation of China (81471073 to HB); National Key R\&D Program of China (2017YFC1309800 and 2017YFC1309801 to HB); Shanghai Municipal Population and Family Planning Commission (201740092 to HY); Special Project of Integrating Traditional Chinese and Western Medicine in Shanghai General Hospital from Shanghai Municipal Population and Family Planning Commission and Shanghai TCM Development Office (ZY(2018-2020)-FWTX-3019 to HY).

Competing interests None declared.

Patient consent for publication Not required.

Ethics approval This study (approval number: B2013-132) was in accordance with the Helsinki Declaration of 1975 and was approved by the ethics committee of Zhongshan Hospital, Fudan University, and each subject provided written informed consent before taking part.

Provenance and peer review Not commissioned; externally peer reviewed.

Data availability statement All data relevant to the study are included in the article or uploaded as supplementary information.

Open access This is an open access article distributed in accordance with the Creative Commons Attribution Non Commercial (CC BY-NC 4.0) license, which permits others to distribute, remix, adapt, build upon this work non-commercially, and license their derivative works on different terms, provided the original work is properly cited, appropriate credit is given, any changes made indicated, and the use is non-commercial. See: http://creativecommons.org/licenses/by-nc/4.0/.

ORCID iD

Hua Bian http://orcid.org/0000-0001-8449-0665

\section{REFERENCES}

1 Doycheva I, Watt KD, Alkhouri N. Nonalcoholic fatty liver disease in adolescents and young adults: the next frontier in the epidemic. Hepatology 2017;65:2100-9.

2 Younossi ZM, Koenig AB, Abdelatif D, et al. Global epidemiology of nonalcoholic fatty liver disease-Meta-analytic assessment of prevalence, incidence, and outcomes. Hepatology 2016;64:73-84.

3 Cohen JC, Horton JD, Hobbs HH. Human fatty liver disease: old questions and new insights. Science 2011;332:1519-23.

4 Musso G, Gambino R, Tabibian JH, et al. Association of nonalcoholic fatty liver disease with chronic kidney disease: a systematic review and meta-analysis. PLoS Med 2014;11:e1001680.

5 Shibata M, Kihara Y, Taguchi M, et al. Nonalcoholic fatty liver disease is a risk factor for type 2 diabetes in middle-aged Japanese men. Diabetes Care 2007;30:2940-4. 
6 Armstrong MJ, Adams LA, Canbay A, et al. Extrahepatic complications of nonalcoholic fatty liver disease. Hepatology 2014;59:1174-97.

7 Wong VW-S, Wong GL-H, Tsang SW-C, et al. High prevalence of colorectal neoplasm in patients with non-alcoholic steatohepatitis. Gut 2011;60:829-36.

8 Li G, Thabane L, Papaioannou A, et al. An overview of osteoporosis and frailty in the elderly. BMC Musculoskelet Disord 2017;18:46.

9 Musso G, Paschetta E, Gambino R, et al. Interactions among bone, liver, and adipose tissue predisposing to diabesity and fatty liver. Trends Mol Med 2013;19:522-35.

10 Kawai M, Rosen CJ. Bone: adiposity and bone accrual-still an established paradigm? Nat Rev Endocrinol 2010;6:63-4.

11 Lee SH, Yun JM, Kim SH, et al. Association between bone mineral density and nonalcoholic fatty liver disease in Korean adults. $J$ Endocrinol Invest 2016;39:1329-36.

12 Cui R, Sheng H, Rui X-F, et al. Low bone mineral density in Chinese adults with nonalcoholic fatty liver disease. Int $J$ Endocrinol 2013;2013:1-6.

13 Pardee PE, Dunn W, Schwimmer JB. Non-Alcoholic fatty liver disease is associated with low bone mineral density in obese children. Aliment Pharmacol Ther 2012;35:248-54.

14 Moon S-S, Lee Y-S, Kim SW. Association of nonalcoholic fatty liver disease with low bone mass in postmenopausal women. Endocrine 2012;42:423-9.

$15 \mathrm{Li} \mathrm{M}, \mathrm{Xu} \mathrm{Y,} \mathrm{Xu} \mathrm{M,} \mathrm{et} \mathrm{al.} \mathrm{Association} \mathrm{between} \mathrm{nonalcoholic} \mathrm{fatty} \mathrm{liver}$ disease (NAFLD) and osteoporotic fracture in middle-aged and elderly Chinese. J Clin Endocrinol Metab 2012;97:2033-8.

16 Bhatt SP, Nigam P, Misra A, et al. Independent associations of low 25 hydroxy vitamin $D$ and high parathyroid hormonal levels with nonalcoholic fatty liver disease in Asian Indians residing in North India. Atherosclerosis 2013;230:157-63.

17 Pacifico L, Bezzi M, Lombardo CV, et al. Adipokines and C-reactive protein in relation to bone mineralization in pediatric nonalcoholic fatty liver disease. World J Gastroenterol 2013;19:4007.

18 Campos RMS, de Piano A, da Silva PL, et al. The role of pro/antiinflammatory adipokines on bone metabolism in NAFLD obese adolescents: effects of long-term interdisciplinary therapy. Endocrine 2012;42:146-56.

19 Purnak T, Beyazit Y, Ozaslan E, et al. The evaluation of bone mineral density in patients with nonalcoholic fatty liver disease. Wien Klin Wochenschr 2012;124:526-31.

20 Pirgon O, Bilgin H, Tolu I, et al. Correlation of insulin sensitivity with bone mineral status in obese adolescents with nonalcoholic fatty liver disease. Clin Endocrinol 2011;75:189-95.

21 Xia M-F, Lin H-D, Yan H-M, et al. The association of liver fat content and serum alanine aminotransferase with bone mineral density in middle-aged and elderly Chinese men and postmenopausal women. J Trans/ Med 2016;14:11.

22 Lima CA, Lyra AC, Rocha R, et al. Risk factors for osteoporosis in inflammatory bowel disease patients. World J Gastrointest Pathophysiol 2015;6:210-8.
23 Bril F, Cusi K. Nonalcoholic fatty liver disease: the new complication of type 2 diabetes mellitus. Endocrinol Metab Clin North Am 2016;45:765-81.

24 Paschou SA, Dede AD, Anagnostis PG, et al. Type 2 diabetes and osteoporosis: a guide to optimal management. J Clin Endocrinol Metab 2017;102:3621-34.

25 Watts NB. Postmenopausal osteoporosis: a clinical review. $J$ Womens Health 2018;27:1093-6.

26 Bhala N, Usherwood T, George J. Non-Alcoholic fatty liver disease. BMJ 2009;339:b2474.

27 European Association for the Study of the Liver (EASL), European Association for the Study of Diabetes (EASD), European Association for the Study of Obesity (EASO). EASL-EASD-EASO clinical practice guidelines for the management of non-alcoholic fatty liver disease. $J$ Hepatol 2016;64:1388-402.

28 Kleiner DE, Brunt EM, Van Natta M, et al. Design and validation of a histological scoring system for nonalcoholic fatty liver disease. Hepatology 2005;41:1313-21.

29 Brunt EM. Histopathology of non-alcoholic fatty liver disease. Clin Liver Dis 2009;13:533-44.

30 Brunt EM, Kleiner DE, Wilson LA, et al. Portal chronic inflammation in nonalcoholic fatty liver disease (NAFLD): a histologic marker of advanced NAFLD-Clinicopathologic correlations from the nonalcoholic steatohepatitis clinical research network. Hepatology 2009;49:809-20.

31 Angulo P, Hui JM, Marchesini G, et al. The NAFLD fibrosis score: a noninvasive system that identifies liver fibrosis in patients with NAFLD. Hepatology 2007;45:846-54.

$32 \mathrm{Kim}$ G, Kim KJ, Rhee Y, et al. Significant liver fibrosis assessed using liver transient elastography is independently associated with low bone mineral density in patients with non-alcoholic fatty liver disease. PLoS One 2017;12:e0182202.

33 Targher G, Lonardo A, Rossini M. Nonalcoholic fatty liver disease and decreased bone mineral density: is there a link? J Endocrinol Invest 2015;38:817-25.

34 Lombardi G, Perego S, Luzi L, et al. A four-season molecule: osteocalcin. updates in its physiological roles. Endocrine 2015;48:394-404.

35 Zoch ML, Clemens TL, Riddle RC. New insights into the biology of osteocalcin. Bone 2016;82:42-9.

36 Greco EA, Francomano D, Fornari R, et al. Negative association between trunk fat, insulin resistance and skeleton in obese women. World J Diabetes 2013;4:31.

37 Pietschmann P, Resch H, Krexner E, et al. Decreased serum osteocalcin levels in patients with postmenopausal osteoporosis. Acta Med Austriaca 1991;18:114-6.

38 Boschitsch EP, Durchschlag E, Dimai HP. Age-Related prevalence of osteoporosis and fragility fractures: real-world data from an Austrian menopause and osteoporosis clinic. Climacteric 2017;20:157-63.

39 Lloyd JT, Alley DE, Hawkes WG, et al. Body mass index is positively associated with bone mineral density in US older adults. Arch Osteoporos 2014;9:175.

40 Starup-Linde J, Vestergaard P. Diabetes and osteoporosis: cause for concern? Eur J Endocrinol 2015;173:R93-9. 